\title{
Optimization of Acetic Acid Production Rate by Thermotolerant Acetobacter spp.
}

\author{
Tahsina Islam', Farzana Diba ${ }^{2}$, Roni Miah', Ayesha Siddiqa1, Nafisa Azmuda1, \\ Shamsun Nahar1, Nihad Adnan1, Shuvra Kanti Dey1, Ali Azam Talukder ${ }^{1 *}$ \\ ${ }^{1}$ Department of Microbiology, Jahangirnagar University, Dhaka, Bangladesh \\ ${ }^{2}$ Department of Microbiology, University of Dhaka, Dhaka, Bangladesh \\ Email: *aat@juniv.edu, *tunubj@yahoo.com
}

How to cite this paper: Islam, T., Diba, F., Miah, R., Siddiqa, A., Azmuda, N., Nahar, S., Adnan, N., Dey, S.K. and Talukder, A.A. (2017) Optimization of Acetic Acid Production Rate by Thermotolerant Acetobacter spp. Advances in Microbiology, 7, 749-759.

https://doi.org/10.4236/aim.2017.711060

Received: October 24, 2017

Accepted: November 21, 2017

Published: November 24, 2017

Copyright $\odot 2017$ by authors and Scientific Research Publishing Inc. This work is licensed under the Creative Commons Attribution International License (CC BY 4.0).

http://creativecommons.org/licenses/by/4.0/

\begin{abstract}
Thermotolerant microorganisms were collected, identified and characterized under different physiological conditions from various rotten fruits in Bangladesh for vinegar production. Among the 15-isolates characterized previously, the strains F-1, F-3 and F-10 represented Staphylococcus, Bacillus and Acetobacter spp., respectively. After checking various parameters for growth, acetic acid production rate was optimized further. Among the 3-starins analyzed here, the strain F-10 gave maximum acetic acid $(7.0 \mathrm{~g} / 100 \mathrm{ml})$ at $37^{\circ} \mathrm{C}$ in $2 \%$ ethanol concentration. The strain F-10 is capable of producing high yield of acetic acid at relatively high temperature, which is an ideal condition for vinegar production, which may reduce the water cooling expenses as well as the risk of contamination.
\end{abstract}

\section{Keywords}

Decomposed Fruits Materials, Thermotolerant Microorganisms, Acetobacter Spp., Acetic Acid, Vinegar Production

\section{Introduction}

Vinegar is a liquid suitable for human consumption produced through the process of fermentation of starch and sugars. It is used in marinating meats, pickling of vegetables, other food-stuffs, and boosts the function of our immune system. According to Food and Drug Administration of the United States (FDA), vinegar contains $4 \%$ solution of acetic acid [1] [2] [3]. Vinegar can be produced by different methods and from various raw materials. The wine (white, red, and sherry wine), cider, fruit musts, malted barley, pure ethanol etc. are used as substrates for vinegar production. 
Acetic acid is the predominant flavoring and antimicrobial component of vinegar. Ethanol can be oxidized to acetic acid by acetic acid bacteria, a large group of obligate aerobic gram negative bacteria [4]. These bacteria are widely distributed in natural habitats and classified into the family Acetobacteraceae. Members of this family are useful in industrial production of vinegar.

Recently the vinegar industry has been developed to produce several types of vinegar using various qualified native or engineered acetic acid bacteria [5] [6]. The demand of acetic acid has been steadily increased because of its use in a variety of industries; however, there has been no significant improvement or major breakthrough in the past decade in producing acetic acid through fermentation. The most likely step to cope with this tremendous demand and predicted shortage of acetic acid appears to be the use of modern microbial production methods. Most of the industries in Bangladesh are making the acetic acid as vinegar produced synthetically instead of microbial fermentation, which is very harmful to health. Bangladesh is a tropical and agro-based country where a huge quantity of cane molasses (about 70,000 tons/year) not only remains unutilized every year but also renders serious environmental pollution. Utilization of this molasses for production of ethanol and then microbial oxidation to acetic acid by indigenous potential bacteria has render attention in recent years. We have identified some strains those could produce acetic acid at high temperature [7]. Among those isolates, for this study we have chosen F-1, F-3, and F-10 strains, as they were the most potent ones according to our previous observation. From those strains, here we have optimized the growth conditions for laboratory scale acetic acid production by Acetobacter spp.

\section{Materials and Methods}

\section{Samples Collection}

Fifteen isolates from decomposed fruits materials were collected for acetic acid production previously and their preliminary characterization were reported [7]. Among these, three isolates (F-1, F-3, F-10) were selected for further analysis to observe their potentiality for acetic acid production under various physiological conditions.

Medium, culture conditions, and cell growth under various physiological environments

Samples were cultured on YPG (Yeast-extract peptone glycerol) plates and Carr medium (3\% yeast extract, ethanol concentration ranging from $2 \%-8 \%$ ) using steak plate methods and incubated at various temperatures $\left(30^{\circ} \mathrm{C}-42^{\circ} \mathrm{C}\right)$ for 4 days to investigate strain's tolerance to high temperature or other stresses. Acetate oxidation was checked with isolated strains from yellow colonies on the agar plate of YPG. Static and shaking culture in above broth medium was also examined and their growth curve were prepared after cells were collected every 4-h interval until 4-days, where necessary. Total cell density was measured at OD $600 \mathrm{~nm}$ by spectrophotometer (Analyticjena, Model-Specord 205, Germany). 


\section{Biochemical Characterization}

Several biochemical tests such as Catalase test, Kligler Iron Agar (KIA) test, Indole test, Nitrate reduction test, Motility, Methyl Red and Voges Proskauer test etc were performed on F-1, F-3, F-10. Biochemical media were inoculated with the pure culture obtained in nutrient agar plates and their biochemical properties were recorded according to the standard guideline [8]. Each test was done in triplicate.

Different sugar fermentation abilities of the isolated strains were examined under identical condition. Experiments were carried out to detect strains capability to degrade various carbon sources in sugar fermentation medium using bromocresol purple as an indicator. Blue color indicates negative and yellow color indicates positive sugar fermentation abilities as shown in respective fields below.

\section{Phylogenetic analysis}

The 16S rRNA products of 15-isolates were amplified from the genome by PCR, purified and sequenced in order to know their strain identity [8]. Forward and reverse sequences were assembled using DNASTAR Lasergene SeqMan software and compared to the GenBank database of the National Center for Biotechnology Information (NCBI) using basic local alignment search tool (BLAST) [9]. To find close phylogenetic relatives, BLAST was performed against NCBI Reference Sequence database [10]. The nucleotide sequences were aligned with CLUSTALW. The phylogenetic analyses were conducted using MEGA version 5 software [11]. Genetic distances were calculated by Kimura two-parameter procedure [12]. Phylogenetic tree was constructed from alignments by the neighbour-joining method and the reliability of inferred trees was tested with bootstrap test.

\section{Estimation of acetic acid production rate}

Each isolate was streaked aseptically on YPG agar plate, incubated overnight at $37^{\circ} \mathrm{C}$. Fresh colony was inoculated into $3 \mathrm{ml}$ of Carr medium and incubated overnight at $37^{\circ} \mathrm{C}$ and the following day re-incubated at $120 \mathrm{rpm}$ in shaking water bath for 0 - 4 days, where necessary. Aeration, inoculums size, glucose content, yeast extract, ethanol etc. were considered as important factors for acetic acid production under the conditions we have employed here. Sample was collected and kept on ice bucket. Acetic acid production rate was estimated by measuring acidity of the culture medium by titration method [9]. In brief, the total amount (in gm) of acetic acid produced in $100 \mathrm{ml}$ of medium was calculated using the following formula:

$$
\begin{aligned}
& \text { Acetic acid }\left(\frac{g}{100} \mathrm{ml}\right) \\
& =\text { Volume of } 0.5 \mathrm{~N} \mathrm{NaOH}(\mathrm{ml}) \text { used in titration } \times 0.03 \times 20
\end{aligned}
$$

\section{Results}

Previously isolated strains, F-1, F-3 and F-10 were selected for this work because; 
they were found to be the most potent ones for acetic acid production among the 15-isolates [7]. Here, they were further characterized phylogenetically based on their 16s rRNA sequence. Apart from that, their biochemical characterizations using various carbon sources, optimal growth and acetic acid production rate in presence of various temperatures, $\mathrm{pH}$, and ethanol concentrations were further analyzed.

\section{Constructions of Phylogenetic Tree}

Phylogenetic tree was prepared based on 16S rRNA sequences. Here, we have selected 3-strains from our 15 isolated strains for genetic analysis, because these were previously found to be the most efficient ones for acetic acid production [1]. After BLAST search, all three isolates (F-1, F-3 and F-10) showed maximum identity of $99 \%$ with the reference strains [7]. Based on phylogenetic tree designed here, F1, F3 and F10 closely resembled Staphyococcus, Bacillus and Acetobacter spp., respectively, and are closely related to each other with acetic acid producing strains as shown in Figure 1 [10] [11]. Therefore, our result revealed that strain F-1, F-3 and F-10 could synthesis acetic acid.

\section{Biochemical Characterization}

Various biochemical tests such as Kligler's Iron Agar (KIA) test, IMViC test, Catalase test, Methyl Red test, Nitrate reduction test and Indole test etc. were performed on selected isolates as shown in Table 1. Our result concludes

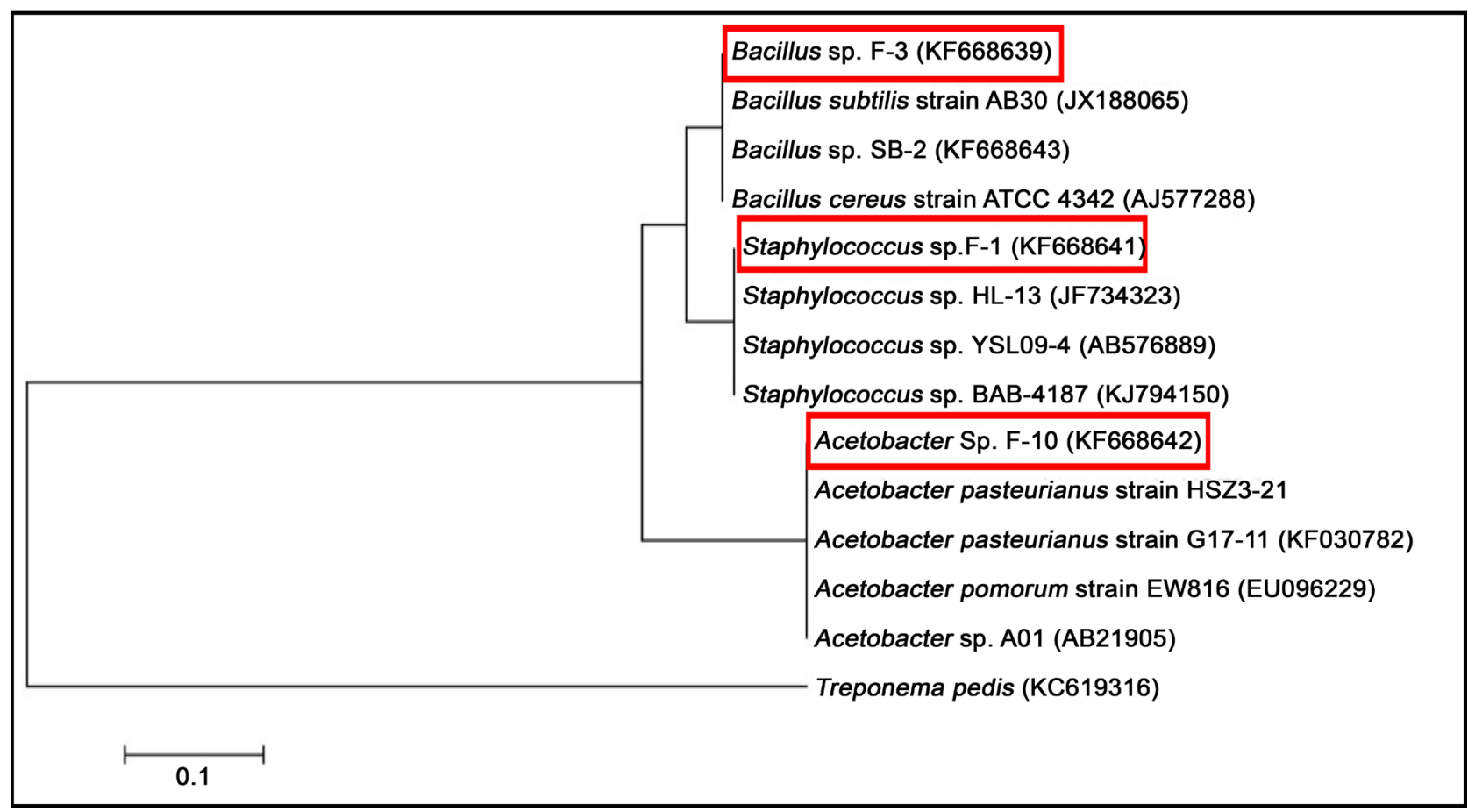

Figure 1. Phylogenetic tree was predicted by the neighbor-joining method using $16 \mathrm{~S}$ rRNA sequences of acetic acid producing microorganisms. The evolutionary distances were computed using the Kimura 2-parameter method and are in the units of the number of base substitutions per site. The bootstrap considered 1000 replicates. The strain Treponema pedis was used as out-group. The scale bar represents the expected number of substitutions averaged over all the analyzed sites. 
Table 1. Biochemical characterization of F-1, F-3 and F-10.

\begin{tabular}{cccccccc}
\hline Sample & Cat & KIA & Cit & VPt & MRt & NRt & Indt \\
\hline F-1 & + & Acidic, Gas & + & + & - & + & + \\
$F-3$ & + & Acidic, Gas & + & + & - & + & + \\
$F-10$ & + & Acidic, Gas & + & + & - & - & + \\
\hline
\end{tabular}

Here, $+=$ Positive and $-=$ Negative. Abbreviations used here are Cat $=$ Catalase test, KIA test, $\mathrm{Cit}=$ Citrate test, $\mathrm{VPt}=$ Voges-Proskauer test, $\mathrm{MRt}=$ Methyl Red test, NRt $=$ Nitrate reduction test and Indt $=$ Indole test. Experiments were done in triplicate.

that all 3-strains could respond various biochemical tests positively with exception of Methyl Red test and Nitrate Reduction test for all and F-10 strain, respectively, which showed negative result.

\section{Effect of sugar fermentation}

Total 14-different sugars (Glucose, Lactose, Sucrose, Galactose, Fructose, Maltose, Mannose, Mannitol, Rhamnose, Starch, Arabinose, Xylose, Xylitol and Sorbitol) were used to check the effect of 3-selected strains on sugar fermentation. It was found that all three isolates (F-1, F-3 and F-10) could ferment most of the sugars as the sole carbon sources and very good fermentation was observed, when media were provided with glucose, fructose or sucrose are shown in Figure 2, and Table 2. On the other hand, all three selected isolates could not ferment Starch, Rhamnose, Arabinose, Sorbitol and Xylose (Figure 2).

\section{Optimization of growth temperature}

Thermotolerant microorganisms could grow well in medium temperatures ranging from $37^{\circ} \mathrm{C}$ to $50^{\circ} \mathrm{C}$ [13]. The growth was monitored by Spectrophotometer at $600 \mathrm{~nm}$. All 3-strains could grow well at tested temperatures ranging from $30^{\circ} \mathrm{C}-42^{\circ} \mathrm{C}$ with some exceptions are shown in Figure 3. The highest and the lowest temperature for cell growth were recorded at $37^{\circ} \mathrm{C}$ and $30^{\circ} \mathrm{C}$, respectively. Among the 3-strains analyzed, the maximum growth was seen in case of strain F-10 (Figure 3). However, we could not observe any significant differences in 4-tested temperatures except $30^{\circ} \mathrm{C}$ (Figure 3, F-10 in bottom panel). The effect of growth temperature on acetic acid bacteria were also checked in various ethanol concentrations ( $2 \%-5 \%)$ for 4 days under shaking condition. All strain could tolerate $2 \%-3 \%$ ethanol concentration. Strain F-3 could grow well at 3\% ethanol concentration rather than $2 \%$ ethanol concentration and the growth was slow and gradually inhibited above $3 \%$ or more ethanol concentrations were used (data not shown).

\section{Estimation of acetic acid production rate}

Finally, acetic acid production abilities were examined by measuring acidity of the culture medium released by acetic acid bacteria used in this study. Cells were cultured in liquid YPG medium together with various ethanol concentrations, incubated at various growth temperatures for 4 days. Acetic acid production rate was estimated by titration method. The maximum and minimum acetic acid content was measured from the strain F-10 and F-01, respectively are shown in Figure 4. Acetic acid amounts were found to be higher and lower in presence of 
$2.0 \%(7.0 \mathrm{~g} / 100 \mathrm{ml})$ and $3.0 \%(5.0 \mathrm{~g} / 100 \mathrm{ml})$ ethanol concentration, respectively (Figure 4). Our result revealed that the strain F-10 showed the maximum acetic acid production rate in $48 \mathrm{~h}$ culture (Figure 4). However, the amount of acetic acid production decreased with the increase of incubation time.

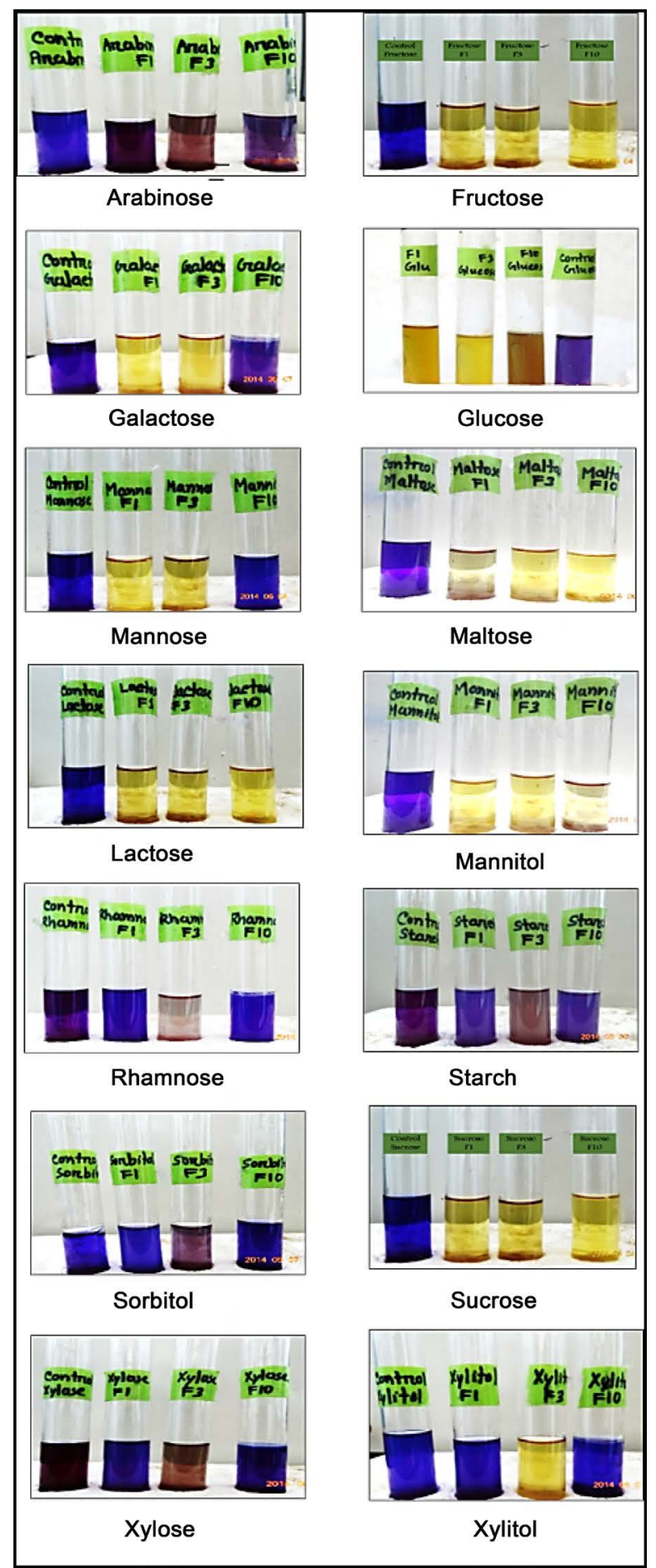

Figure 2. Different sugar fermentation abilities of isolated strains F-1, F-3 and F-10. Experiment was carried out to observe strains capability to ferment various sugars in fermentation medium. Experiments were done in triplicate. 

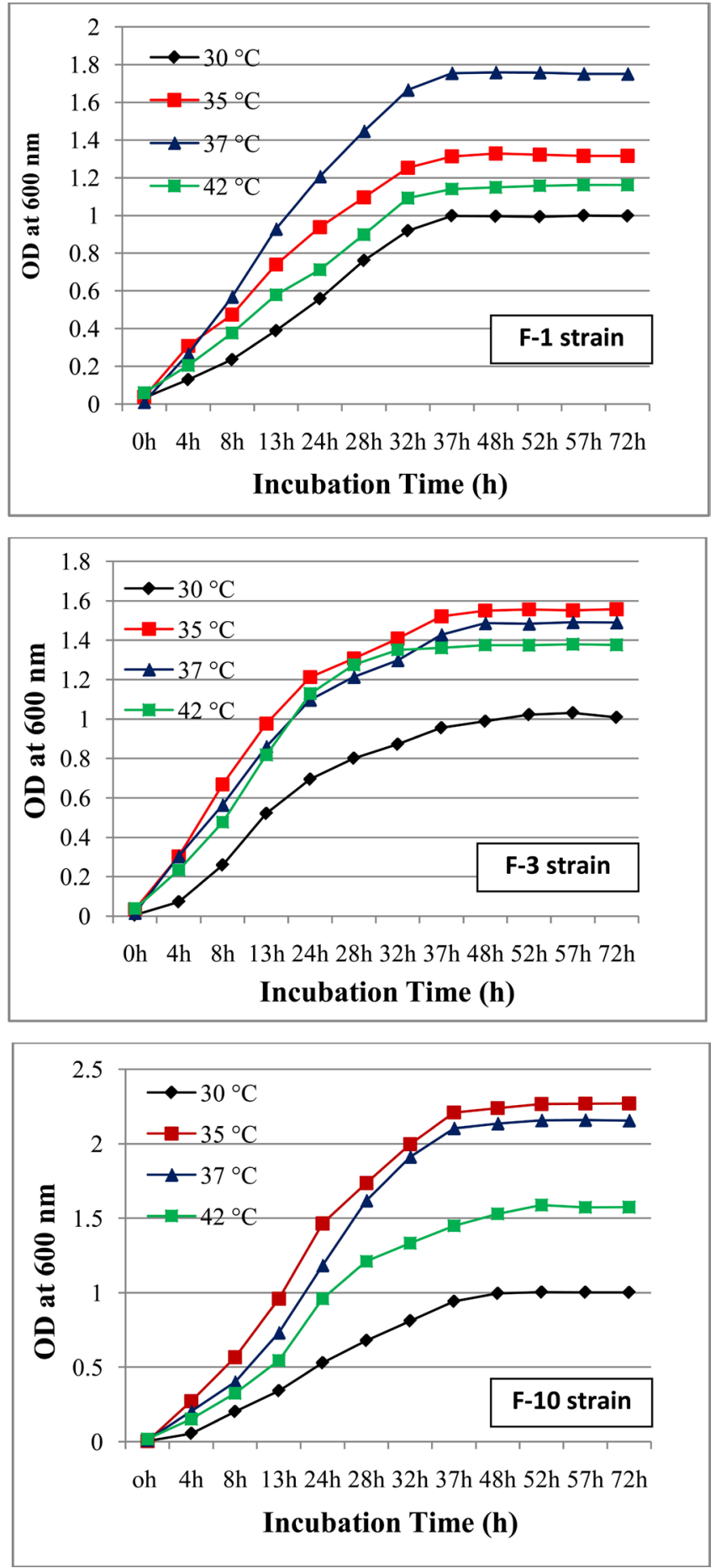

Figure 3. Growth curve of three selected strains at different temperatures. Top, middle and bottom represent the growth curve pattern of strains F-1, F-3 and F-10, respectively. Fresh single colony was inoculated into YPEG broth and incubated overnight at $37^{\circ} \mathrm{C}$. Fresh culture of each 3-strains was diluted 500-fold into the fresh medium and incubation was continued until $72 \mathrm{~h}$ at 4 -different tested temperatures are shown. Samples were collected in every $4 \mathrm{~h}$-inteval and put them on ice bucket for seize the growth and then their growth were measured by spectrophotometer (Specord UV/Visible Spectrophotometer, Analytic jena, Germany) at $600 \mathrm{~nm}$ against the YGEA broth as blank. The experiments were carried out in triplicates and the mean values are used here. 

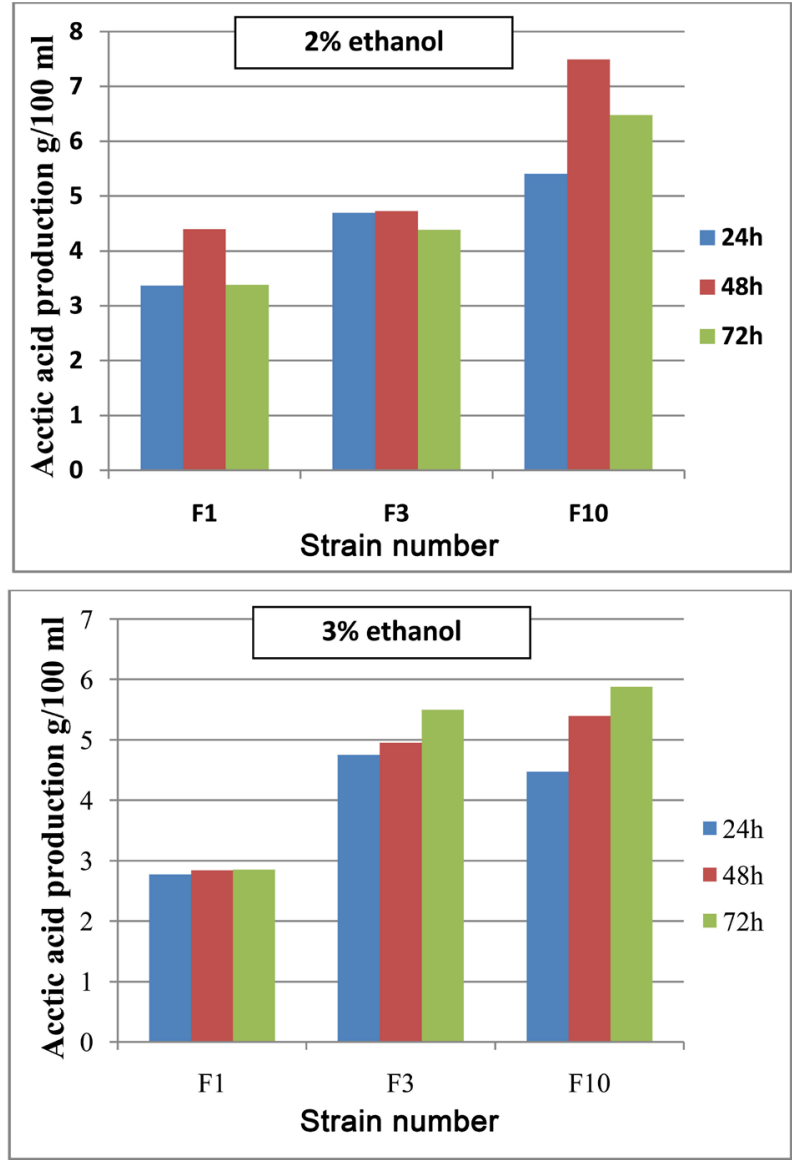

Figure 4. Effect of ethanol concentration on acetic acid production. Here, upper and bottom panel represent the acetic acid production rate in $2 \%$ and $3 \%$ ethanol concentrations, respectively. Cells were cultured in liquid YPG medium at $37^{\circ} \mathrm{C}$ in various indicated time point. Samples were collected and put on ice bucket for seize the growth and then their acetic acid production rate was estimated by titration method are explained in Materials and Methods. The experiments were carried out in triplicates and the mean values are used here.

\section{Discussion}

Vinegar production requires acetic acid bacteria that produce, tolerate and conserve high levels of acetic acid. We have isolated several strains from the natural decomposed fruit materials, which could produce acetic acid under high temperature fermentation process [7]. In this study, our main target was to optimize acetic acid production rate by stress tolerant as well as thermotolerant Acetobacter spp. which was isolated from the natural fermented sources in Bangladesh for vinegar production under different physiological conditions.

Most Acetobacter strain utilized different carbohydrates or sugars during acetic acid production [14]. Fourteen carbon sources were used for checking the fermentation abilities of the tested 3-isolated (Figure 2). Under the conditions we have employed here, all isolates were able to oxidize about half of tested sugars (Table 2). This result revealed that some strains could utilize special pathway for sugar fermentation during vinegar production, which have to be clarified in future. 
Table 2. Fermentation effect of various carbon sources on F-1, F-3 and F-10.

\begin{tabular}{cccccccccccccccc}
\hline Sample & Lac & Glu & Suc & Fru & Ara & Mann & Xyl & Mant & Rham & Sorb & Mal & Gal & Starch & Xylt \\
\hline$F-1$ & + & + & + & + & - & $+/-$ & - & + & - & - & + & + & - & + \\
$F-3$ & + & + & + & + & - & $+/-$ & - & + & - & - & + & + & - & + \\
$F-10$ & + & + & + & + & - & $+/-$ & - & + & - & - & + & - & - & \\
\hline
\end{tabular}

Here, $+=$ Positive and $-=$ Negative.$+-=$ Variable fermentation effect. Abbreviations used here are Lac $=$ Lactose, $\mathrm{Glu}=$ Glucose, Suc $=$ Sucrose, Fru $=$ Fructose, Ara $=$ Arabinose, Mann $=$ Mannose, $\mathrm{Xyl}=$ Xylose, Mant $=$ Mannitol, Rham $=$ Rhamnose, Sorb $=$ Sorbitol, Mal $=$ Maltose, Xylt $=$ Xylitol . All the strains showed positive results for lactose, Glucose, Sucrose, Fructose, Mannitol, Maltose and none of them could degrade Arabinose, Xylose, Rhamnose, Sorbitol and Starch in sugar fermentation medium using bromocresol purple as an indicator. Here, blue color indicates negative and yellow color indicates positive activity. Experiments were done in triplicate.

In addition to various sugars, the medium $\mathrm{pH}$, temperature and ethanol concentrations are also considered as important physiological factors to accelerate growth of microorganisms and to reduce chance of contamination for the high yield of acetic acid production. Most of Acetobacter strains can effectively grow between $\mathrm{pH} 4.5$ - 7.0 [14]. We have checked the various medium $\mathrm{pH}$ (3.0, 4.0, 5.0, 6.0, 7.0 and 8.0) in YGEA broth until 72 hours of growth. The maximum growth was observed within the $\mathrm{pH} 5.0$ - 6.0 (data not shown). Our results are consistent with the result published previously found that the optimal $\mathrm{pH}$ for the growth of acetic acid bacteria (AAB) is between $\mathrm{pH} 5.0$ - 6.0 [3]. Among the various growth temperatures, $\mathrm{pH}$ and ethanol concentrations were checked, and we concluded that the optimum temperature, $\mathrm{pH}$ and ethanol concentration was found to be $37^{\circ} \mathrm{C}, 6.0$ and $2.0 \%$ ethanol, respectively, which gave $7.0 \mathrm{~g} / 100 \mathrm{ml}$ acetic acid (Figure 4). Our results are consistent with the result published previously by $\mathrm{Lu}$ et al. [15].

The higher the concentration of ethanol present in the medium, the more acetic acid could be produced. But again, microorganisms cannot withstand higher concentration of ethanol in their growth medium. Because of these reasons, we choose various concentrations of ethanol $(2 \%-3 \%)$ in our test media, and optimized the condition for highest acetic acid production by our strains. Strain F-10, the isolated Acetobacter, could produce remarkable amount of acetic acid even in $3.0 \%$ or above ethanol concentration (Figure 4). Other 2-strains grow well at $2.0 \%$ ethanol concentration but growth rate was decreased gradually when increased ethanol concentration $3.0 \%$ or above (Figure 4). This might happened due to excess formation of acetic acid by consuming sugar in the fermentation medium. Thus further inhibiting the acetic acid production rate which control growth at relative high ethanol concentration (Figure 4). On the other hand, over-oxidation is another serious problem during vinegar production in tropical and temperate countries [16]. The main reason of over-oxidation is uncontrolled temperature was applied during vinegar preparation. In addition, changes in the medium microbial population or in the strain physiology, as a result of the lack of the alcoholic substrate are also considered the reasons for over-oxidation [16]. 
In addition to F-10 (Acetobacter spp), F-1, encoding Bacillus spp. could also produce acetic acid or its derivatives [17]. However, till date no report is available for Staphylococcus spp., to produce acetic acid. Moreover, it was reported previously that acetic acid can induce Staphylococcus spp gene expression [18]. Based on phylogenetic tree designed here, the strain F-3 is closely related to other acetic acid producing strains [10] [11]. Therefore, under the conditions we have employed here, our result revealed that F-3 could also synthesis acetic acid which has to be clarified more in future.

\section{Conclusion}

Among the three strains (F-1, F-3 and F-10) analyzed here, we have concluded that the thermotolerant strain F-10 (Acetobacter spp) produced maximum amount $(7.0 \mathrm{~g} / 100 \mathrm{ml})$ of acetic acid in presence of $2 \%$ ethanol in the medium.

\section{Acknowledgements}

Authors are grateful to Prof. K. Matsushita and Prof. M. Yamada (Yamaguchi University, Japan) for providing DNA sequence facilities. The research work was financially supported by HEQEP (W1, cp-3413), Ministry of Education, Government of the Peoples Republic of Bangladesh.

\section{References}

[1] Joyeux, A., Lafon-Lafourcade, S. and Ribereau-Gayon, P. (1984) Evolution of Acetic Acid Bacteria during Fermentation and Storage of Wine. Applied Environmental Microbiology, 48, 153-156.

[2] Drysdale, G.S. and Fleet, G.H. (1988) Acetic Acid Bacteria in Wine-Making: A Review. American Journal of Enology and Viticulture, 39, 143-154.

[3] Kocher, G.S., Kalra, K.L., and Phutela, R.P. (2006) Comparative Production of Sugarcane Vinegar by Different Immobilization Techniques. Journal of the Institute of Brewing, 112, 264-266. https://doi.org/10.1002/j.2050-0416.2006.tb00722.x

[4] Saha, P. and Banerjee, S. (2013) Optimization of Process Parameters for Vinegar Production Using Banana Fermentation. International Journal of Research in Engineering and Technology, 2, 501-514. https://doi.org/10.15623/ijret.2013.0209076

[5] Drysdale, G.S. and Fleet, G.H. (1989) The Growth and Survival of Acetic Acid Bacteria in Wines at Different Concentrations of Oxygen. American Journal of Enology and Viticulture, 40, 99-105.

[6] Harada, T. and Mori, H. (1971) Mutants of Acetic Acid Bacteria Used in Vinegar Production. Journal of Fermentation Technology, 49, 836-841.

[7] Diba, F. Alam, F. and Talukder, A.A. (2015) Screening of Acetic Acid Producing Microorganisms from Decomposed Fruits for Vinegar Production. Advances in Microbiology, 5, 291-297. https://doi.org/10.4236/aim.2015.55028

[8] Garrity, G.M., Brenner, D.J., Krieg, N.R. and Staley, J.T. (2005) Bergey’s Manual of Systematic Bacteriology. Sec. Ed., Vol. 2, Springer, New York, 41-79.

[9] Beheshti Maal, K. and Shafiei, R. (2010) Isolation and Characterization of an Acetobacter Strain from Iranian White-Red Cherry as a Potential Strain for Cherry Vinegar Production in Microbial Biotechnology. Asian Journal of Biotechnology, 1, 53-59. 
[10] Pruitt, K.D., Tatusova, T. and Maglott, D.R. (2005) NCBI Reference Sequence (RefSeq), A Curated Non-Redundant Sequence Database of Genomes, Transcripts and Proteins. Nucleic Acids Research, 33, 501-504. https://doi.org/10.1093/nar/gki025

[11] Tamura, K., Dudley, J., Nei, M. and Kumar, S. (2007) MEGA4: Molecular Evolutionary Genetics Analysis (MEGA) Software Version 4.0. Molecular Biology Evolution, 24, 1596-1599. https://doi.org/10.1093/molbev/msm092

[12] Kimura, M.A. (1980) Simple Method for Estimating Evolutionary Rates of Base Substitutions through Comparative Studies of Nucleotide Sequences. Journal of Molecular Evolution, 16, 111-120. https://doi.org/10.1007/BF01731581

[13] Talukder, A.A., Easmin, F., Mahmud, S.A. and Yamada, M. (2016) Thermotolerant Yeasts Capable of Producing Bioethanol: Isolation from Natural Fermented Sources, Identification and Characterization. Biotechnology \& Biotechnological Equipment, 2818, 1-9. https://doi.org/10.1080/13102818.2016.1228477

[14] Hassan, M., Diaz, R., Rosalinda, A.M., Othman, N., Ramlian, A., Hesham, A. and Enshsay, E.L. (2012) Efficient Production Process for Food Grade Acetic Acid by Acetobacteraceti in Shake Flask and in Bioreactor Cultures. Journal of Chemistry, 9 , 2275-2286.

[15] Lu, S., Lee, F. and Chen, H. (1999) A Thermotolerant and High Acetic Acid-Producing Bacterium Acetobacter sp. I14-2. Journal of Applied Microbiology, 86, 55-62. https://doi.org/10.1046/j.1365-2672.1999.00633.x

[16] Saeki, A., Taniguchi, M., Matsushita, K., Toyama, H., Theeragool, G., Lotong, N. and Adachi, O. (2014) Microbiological Aspects of Acetate Oxidation by Acetic Acid Bacteria, Unfavorable Phenomena in Vinegar Fermentation. Bioscience Biotechology and biochemistry, 61, 138-145.

[17] Silva, A.L.L. and Costa, J.L.U.Z. (2015) Production of Indole-3-Acetic Acid by Bacilluss spp Isolated from Different Soils. Bulgarian Journal of Agricultural Science, 21, 282-287.

[18] Rice, K.C., Nelson, J.B., Patton, T.G., Yang, S., Bayles, K.W. and Al, R.E.T. (2005) Acetic Acid Induces Expression of the Staphylococcus aureus cidABC and $\operatorname{lrg} A B$ Murein Hydrolase Regulator Operons. Journal of Bacteriology, 187, 813-821. https://doi.org/10.1128/JB.187.3.813-821.2005 